\title{
Due antichi quartieri romani sotto la lente di ingrandimento: un'analisi space syntax dei quartieri delle città antiche e della loro vita sociale
}

\author{
Two ancient city blocks under the magnifying glass: a space syntax analysis \\ of Ostia's urban quarters and their social life
}

\section{Dos bloques urbanos bajo la lente de aumento: un análisis sintáctico-espacial de los barrios urbanos de Ostia y su vida social}

\author{
Hanna Stöger \\ University of Leiden \\ e-mail: h.stoger@arch.leidenuniv.nl \\ Eraldo Brandimarte \\ Knevel Architecten \\ e-mail: eraldo.brandimarte@gmail.com
}

\begin{abstract}
RESUMEN
El estudio de los vecindarios y el uso social del espacio habitado son temas de interés creciente. Mediante la combinación de métodos arqueológicos y space syntax, este artículo ofrece nuevas formas de analizar el entorno físico de la vida cotidiana en las ciudades romanas. El examen detallado de dos bloques urbanos en Ostia (la ciudad portuaria de Roma en época imperial) nos ha permitido identificar espacios que fomentan la cohesión social y la vida en comunidad. El uso común de patios y pasillos en el Bloque IV ii sugiere un uso comunal continuado desde el siglo II hasta el siglo V d. C. En claro contraste con este sector, el Bloque IV iv no tiene espacios comunes y muestra edificios aislados con accesos individuales al espacio público. El Bloque IV ii disfruta de espacios compartidos dentro de su propio perímetro, mientras que el Bloque IV iv parece estar orientado por el interés de comunidades externas con actividades desarrolladas hacia la calle creando cierto aislamiento del bloque. Estos resultados muestran la flexibilidad de las estructuras urbanas romanas y nos permiten atisbar la actividad de comunidades que permitieron el desarrollo sostenible de la ciudad en procesos de larga duración.
\end{abstract}

Palabras clave: Arqueología romana; Ostia; ciudades portuarias; space syntax; vecindarios urbanos; vida en comunidad.

\footnotetext{
ABSTRACT

Neighbourhoods and the social use of space are areas of growing interest. By combining archaeological methods and space syntax, the article offers new insights into the physical environment of daily life in the Roman city. The detailed examination of two city blocks from Ostia (Rome's imperial port-town), allows the authors to identify spaces which fostered social cohesion and community building. The shared courtyards and passage spaces of Block IV ii suggest continued community focus (2nd - 5th centuries AD). In contrast, Block IV iv lacks common spaces and revealed self-contained buildings centred on individual access to public space. Block IV ii enjoyed shared spaces within its own perimeter, while Block IV iv looks toward external community building, with activities centred on the street confining the block. The results show the flexibility of Roman urban structures and allow for suggestive glimpses into the community that sustained the city in the long-term.
} 
Keywords: Roman archaeology; Ostia; port-towns; space syntax; urban neighbourhoods; community building.

Recibido: 19-05-2015. Aceptado: 07-10-2015.

Cómo citar este artículo / Citation

Fernández García, G. 2015: "La articulación del espacio doméstico en las casas de patio central. Un estudio para el Noreste peninsular ibérico entre los siglos IV - II a. C.", Arqueología de la Arquitectura, 12: e033. doi: http://dx.doi.org/10.3989/arq.arqt.2015.123

\section{Copyright}

(c) 2015 CSIC. Este es un artículo de acceso abierto distribuido bajo los términos de la licencia Creative Commons Attribution-Non Commercial (by-nc) Spain 3.0.

\section{INTRODUZIONE}

La cultura Romana può essere considerata come una cultura essenzialmente urbana, basti considerare come a metà del II sec. d.C. l'Impero romano contava ben più di 2000 insediamenti urbani. Intensive indagini sulle città romane meglio preservate (come ad esempio Pompei, WallaceHadrill 1994; Allison 2004; Laurence 2007) e studi comparativi ad ampio raggio su tutto l'Impero, hanno ampliato la nostra conoscenza dell'urbanistica romana e delle sue implicazioni economiche e socio-politiche (ad esempio Bowman e Wilson 2011; Laurence, Esmonde Cleary e Sears 2011). Tuttavia nella maggior parte dei casi questi studi non sono stati condotti nel dettaglio dandoci così ben poche informazioni riguardo l'immediata, reale e concreta vita quotidiana degli abitanti delle città antiche. La ricerca in corso nell'ambito di Neighbourhoods of Roman Ostia ha l'obiettivo di rimediare a questo sbilanciamento e nello stesso tempo mira ad estendere ed integrare la nostra attuale conoscenza delle città antiche attraverso un approfondito e localizzato studio dei quartieri di Ostia, l'antica città portuale di Roma ${ }^{1}$. Queste unità urbane costituiscono un campione ben rappresentativo dell'ambiente fisico nel quale si svolgeva la vita quotidiana degli antichi abitanti di Ostia. Presumibilmente quest'ambiente edifificato condiviso, che vedeva gli antichi residenti vivere in notevole prossimità e vicinanza, deve aver avuto un forte impatto nella loro vita sociale ed economica. E' in ogni caso possibile parlare a pieno titolo di quartieri urbani antichi o ci confrontiamo con specifici fenomeni culturali che differiscono dalla concezione contemporanea di una ben funzionante blocco cittadino? Per trovare una risposta a questo quesito la ricerca offre una accurata lettura archeologica e spaziale

\footnotetext{
${ }^{1}$ Il post-dottorato di ricerca nell'ambito dei 'qurtieri urbani ostiensi' viene portato avanti dall'autore presso l'Università di Leiden, nei Paesi Bassi, con la gentile collaborazione e autorizzazione della Sopraintendenza Speciale per i Beni Archeologici di Roma, sede di Ostia.
}

dello spazio urbano ostiense del passato, concentrandosi su due dei suoi blocchi cittadini, il IV ii ed il IV iv (fig.1). La complessità dei resti archeologici e degli interrogativi sociali che sono sostanza di questa ricerca richiedono l'applicazione di una combinazione di diverse metodologie. Queste includono una valutazione ed una documentazione archeologica accurate delle strutture pervenuteci, un esame critico del materiale pubblicato e non (materiale d'archivio) e soprattutto un'analisi space syntax dell'organizzazione spaziale dei blocchi cittadini. Le teorie ed i metodi della space syntax sono di fondamentale importanza per questo studio in quanto consentono di andare oltre allo statico dato archeologico (ad esempio l'ambiente costruito del passato) e permettono una comprensione delle dinamiche sociali di queste aree urbane che non sarebbe altrimenti possibile (Hillier e Hanson 1984; Hillier 1996; Hillier e Vaughan 2007). L'obiettivo di questa ricerca sui quartieri ostiensi é quello di identificare fattori spaziali che possano aver contribuito alla creazione e alla promozione di una coesione sociale e di una comunità di quartiere, quanto quello di verificare come la vitalità del quartiere possa aver costituito la causa della suo continuo utilizzo in un lungo arco di tempo, sia a livello locale che cittadino.

\section{SPACE SYNTAX E ARCHEOLOGIA}

Se da una parte la space syntax gode di costantemente crescente popolarità nel campo dell'archeologia (Vedi per esempio Paliou, Lieberwirth e Polla 2014, e anche Bermejo 2014), nello stesso tempo, tra tutte le scienze umanistiche l'archeologia gode di una posizione eccezionale e privilegiata agli occhi della space syntax dal momento che essa si occupa più delle altre di "spazio reale". La space syntax percepisce l'archeologia come una disciplina intrinsecamente spaziale, il cui interesse per lo spazio affonda le sue radici in profondità nella sua tradizione ed é costantemente rinnovato dalla sua pratica (Hillier 2008: 223). 


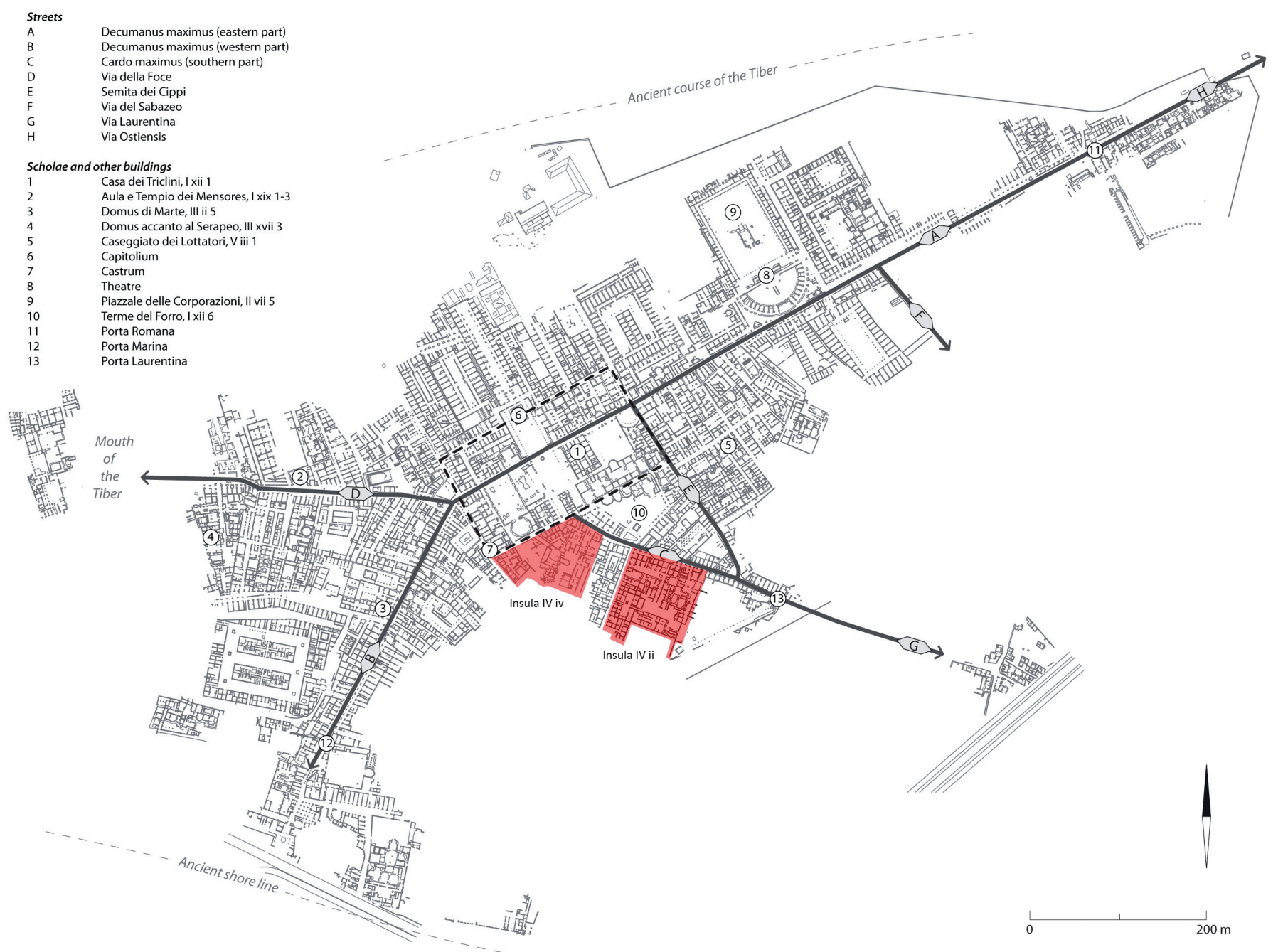

Figura 1. Pianta del Sito Archeologico di Ostia Antica con l'indicazione dei quartieri urbani (blocchi cittadini) oggetto dell'analisi archeologica e spaziale (IV ii, iv). La nomenclatura IV ii, iv fa riferimento alla posizione di questi blocchi cittadini nell'ambito degli scavi di Ostia.

Nonostante questo reciproco apprezzamento e riconoscimento il rapporto tra l'archeologia e la space syntax non è interamente privo di problematicità, portando l'archeologia a confrontarsi con nuove sfide teoriche e metodologiche. Nell'arco degli ultimi 30 anni l'archeologia ha incorporato con successo vari approcci teoretici e metodologici definiti "analisi spaziali". Tuttavia, come sottolineta da Hillier (2008: 223), molti di loro sembrano condividere il presupposto che lo spazio acquisisce significato solo attraverso un agente esterno o un processo sociale che gli attribuisce senso e forma. Da ciò consegue che le forme spaziali dovrebbero essere studiate solo in relazione alle loro cause sociali. Questo ruolo predominante attribuito all'intervento umano nelle trasformazioni spaziali porta ad un problema metodologico in archeologia, privando la cultura materiale, nel nostro caso forme spaziali fisiche, di qualsiasi influenza attiva sulla vita dell'uomo. D'altro canto però la pratica archeologica costituisce la conferma che la conoscenza del passato può essere recuperata da forme prettamente materiali della vita quotidiana di chi ci ha preceduto. Per questo motivo quindi le forme materiali dovrebbe essere di centrale interesse e meritano di essere studiate approfonditamente, rendendo lo spazio urbano del passato di per sé un oggetto di indagine ed un elemento di interesse teorico.

\section{LA SCELTA DI QUARTIERI ANTICHI}

I quartieri costituiscono un oggetto di interesse e studio sia per gli addetti alla pianificazione urbana contemporanea sia per gli archeologi. Questi ultimi si concentrano sullo spazio costruito del passato e possono offrire una 
prospettiva a lungo termine sulle direzioni e su i modelli dello sviluppo urbano (Vedi sopratutto Smith 2010b: 229230). La qualità dei quartieri urbani è da tempo riconosciuta come il principale indicatore della stabilità residenziale e dello sviluppo delle città (Vedi per esempio Gans 1962). I quartieri ricoprono un ruolo cruciale nella vita quotidiana degli abitanti della città; non solo ne costituiscono la realtà fisica di vicinanza spaziale ma creano inoltre un sentimento di appartenenza in grado di generare sentimenti di stabilità e sicurezza. Quartieri rigidamente organizzati in maglie troppo strette, ad esempio, possono essere vissuti come comunità chiuse che esercitano un forte controllo sociale su i propri residenti, complicando l'integrazione dei nuovi arrivati i quali difficilmente si sentiranno parte di uno spazio condiviso. Quartieri ben funzionanti, d'altra parte, sono forieri di una grande quantità di benefici personali e sociali. Questi creano sicurezza per le persone e le loro proprietà in quanto i residenti vigilano per gli altri residenti e le loro abitazioni. Una vivace unità di quartiere può stabilire utili e benefici legami sociali tra i suoi abitanti, tra i residenti, tra i servizi locali e gli esercizi commerciali, promuovendo nello stesso tempo una maggior cura per gli spazi pubblici e privati in generale (cf. Sampson, Morenoff e Gannon-Rowley 2002). I quartieri contribuiscono allo sviluppo della comunità e possono agire da punto focale per l'avvio e l'attuazione dei cambiamenti sociali (Moulaert, Martinelli, Swyngedouw e Gonzalez 2013: 5).

I recenti studi archeologici denotano un crescente interesse per i quartieri ed il loro studio, sia in ambito urbano che rurale, dall'antichità attraverso i vari periodi storici. I quartieri sono stati oggetto di approfondita analisi in contesti quali quello di Pompeii (Laurence 2007; Jones e Robinson 2007), della Roma Augustea (Bert Lott 2004), delle città mesoamericane di Teotihuacan e Tikal (Arnauld, Manzanilla e Smith 2012), dell'Anatolia (Catalhöyük) (Düring 2006) e della Mesopotamia, ad esempio Nippur (Stone 1987). Negli ultimi decenni gli studi archeologici hanno spostato la loro attenzione dall'elitario e monumentale alle vite quotidiane dei comuni abitanti della città. Studiare le città antiche a scala di quartiere consente di far luce non solo sulle attività di tutti i giorni dei suoi abitanti ma anche sull'interpretazione funzionale dei diversi edifici che la costituiscono. Le città romane in particolare costituiscono un buon campo di ricerca per gli studi sui quartieri in quanto offrono un numero sostanziale di resti architettonici ben preservati corredati da una grande ricchezza di testimonianze testuali riguardanti la vita quotidiana (per esempio gli epigrammi di Marziale e le satire di Orazio). La maggior parte di queste testimonianze scritte tuttavia riguardano essenzialmente la città di Roma, testi simili in riferimento alla vita quotidiana ostiense infatti sono pressochè assenti. D'altra parte l'ambiente costruito del passato di Ostia costituisce una migliore evidenza archeologica della vita quotidiana romana, in quanto queste evidenze archeologiche nella città di Roma rimangono in gran parte oscurate dalla densità degli insediamenti urbani e dalla sporadicità degli scavi.

Nell'ambito di questo studio un quartiere viene definito come una porzione della città nella quale i suoi abitanti vivono le loro vite quotidiane. Ciò implica che $\mathrm{i}$ quartieri sono caratterizzati da stretti legami tra i propri abitanti, spesso scanditi da quotidiani interazioni e contatti diretti. Gli studi spaziali nello specifico identificano un quartiere in base alla sua capacità di instaurare relazioni tra i suoi residenti attraverso l'impianto architettonico (Suttles 1972 in Smith 2010a: 139), alla presenza di servizi condivisi quali fontane d'acqua (cf. Laurence 2007: 39-61), negozi o altre necessità quotidiane (Van Nes 2011) e, nel caso della nostra città romana, santuari locali o tempi a servizio di una particolare comunità.

I blocchi della città antica presi in considerazione per questo studio rappresentano spazialmente una terra di mezzo e agiscono da filtro mediando fra l'unità familiare individuale e la più ampia città. Ciò pone il quartiere nel punto d'intersezione tra le necessità dei residenti e le esigenze infrastrutturali imposte dalla città. Questa natura dicotomica, nello stesso tempo locale e globale, lancia una sfida agli approcci tradizionali dell'archeologia, troppo spesso concentrati solo sugli aspetti locali delle unità urbane rinnegando la loro relazione con il contesto generale cittadino (per esempio Boersma 1985; Gering 2002). Lo studio qui presentato, nel suo approccio ai quartieri, combina la prospettiva archeologica con quella spaziale analizzandoli sia come entità locali che come parti costituenti del più ampio paesaggio urbano. Questo obiettivo viene raggiunto valutando l'organizzazione intera dei blocchi cittadini ed il loro livello di integrazione con la rete stradale urbana, esaminando il loro grado di accessibilità agli edifici pubblici, ai luoghi e a i servizi offerti dalle infrastrutture della città.

\section{UN DIALOGO TRA CITTÀ ANTICA E CITTÀ MODERNA}

La diffusione globale dei quartieri suggerisce la presenza di una sottesa struttura formale che consente ai 
ricercatori di valutarne e misurarne oggettivamente l'organizzazione nel tempo (Dalton 2006; Smith 2010a). Questo studio sui blocchi cittadini di Ostia è stato ispirato dai progressi ottenuti dalla ricerca sui quartieri contemporanei (ad esempio Vaughan, Jones, Griffiths e Haklay 2010) e dal crescente interesse nell'organizzazione spaziale delle città del passato (Laurence 2007; Smith 2010a; Stöger 2011; Scott 2012). I recenti studi sul paesaggio urbano ostiense, in particolare il lavoro di Medri e Di Cola (2013) sulle Terme del Nuotatore all'interno dell'Insula V, hanno anche cominciato a porre le considerazioni spaziali in cima alla loro agenda di ricerca. I blocchi cittadini nelle città Romane sono solitamente denominati Insulae, gruppi di edifici spesso delimitati da strade sui quattro lati o in altro modo chiaramente separati dalle insulae circostanti, e costituisono essenzialmente il tessuto della città Romane.

Lo studio dei quartieri ostiensi ha l'ambizione di istaurare un dialogo tra i quartieri urbani antichi e moderni combinando metodi archeologici a tecniche analitiche utilizzate oggi dalle discipline urbanistiche. Varie metodologie di analisi spaziale sono state sviluppate per far fronte alle problematiche poste dalla pianificazione urbana contemporanea. Sebbene inizialmente sviluppate per la pianificazione urbana contemporanea, le teorie ed i metodi dalla space syntax sono stati utilizzati con successo da archeologi per lo studio delle città romane (Laurence 2007; Grahame 2000; Kaiser 2000, 2011; Stöger 2011). L'applicazione degli stessi metodi allo studio delle città antiche e moderne mira ad identificare un linguaggio comune nello studio dell'urbanistica, riconoscendo il potenziale dell'archeologia di prefigurare sviluppi a lungo termine ed individuare tendenze o modelli temporali dell'evoluzione delle città (Vedi Smith 2010b).

\section{QUARTIERI INTERESSATI}

Ostia, la principale città portuale di Roma, offre un promettente campo di verifica per le indagini riguardanti l'ambiente costruito del passato. L'esteso e ben preservato paesaggio urbano gode delle condizioni ideali per investigare sulla capacità ed il potenziale del contesto architettonico di generare e possibilmente anche sostenere lo sviluppo dei quartieri urbani in un ampio arco temporale. La lunga e continua storia della città-porto è la testimonianza di un millennio di cultura urbana Romana. Diverse etnie, religioni e culture da tutta l'area mediterranea interagivano in questo dinamico contesto portuale, generando un ambiente particolarmente sensibile, ricettivo dei cambiamenti in atto nel più ampio mondo Romano. I quartieri urbani hanno così un duplice carattere, essendo simultaneamente discrete entità locali e componenti urbane dotate di un impatto attivo sull'intera città.

\section{INSULAE DI OSTIA ESAMINATE NEL DETTAGLIO}

I blocchi cittadini ostiensi forniscono una prospettiva sullo sviluppo dei quartieri nell'arco di un periodo temporale che va dal periodo Repubblicano (II sec. a.C.) fino a quello Tardo Antico (V e VI sec. d.C.). I blocchi dell'insula scelti per un'analisi approfondita si trovano in diverse aree della città, questi differiscono per composizione architettonica e spaziale seppur coprendo stessi periodi di occupazione (dal periodo Tardo Repubblicano al V sec. d.C.). Se da una parte i dati archeologici e spaziali sono abbondantemente disponibili per il blocco IV ii (Stöger 2011), dall'altra il blocco IV iv sino ad ora ha attratto solo limitatamente l'attenzione degli studiosi (Lorenzatti 1998; Guidobaldi 1995) e rimane per larga parte non approfondito ne pubblicato. L'analisi spaziale qui presentata si basa su una completa valutazione archeologica portata avanti dall'autore nell' arco di diversi anni (Stöger 2011; Terpstra $2014^{2}$ ). Il lasso temporale d'interesse per questo studio è la prima metà del III sec. D.C. (Età Severiana); durante questo periodo tutti gli edifici esistenti nel blocco IV ii erano in uso, mentre il blocco IV iv sono stati oggetto di attività di riorganizzazione e sviluppo. Nell'ambito della lunga storia di Ostia, selezionare la prima metà del III secolo per la nostra ricerca significa porre la discussione spaziale tra dui grandi fasi di sviluppo urbano: l'espansione urbana della città nel corso II sec. d.C. e all'inizio del III sec. la trasformazione da perno commerciale focalizzato verso l'esterno a città che risponde alle necessità di una crescente clientela locale (Stöger 2011: 160).

\section{BLOCCO CITTADINO IV II. UN QUARTIERE PER DEFINIZIONE}

Un precedente studio sull'Insula IV ii (Stöger 2011, 2014) si è basato sui concetti spaziali ed ha applicato

\footnotetext{
2 Terpstra, D. 2014: Space and Neighbourhoods in Roman Ostia: The Spatial Organisation of Ostia's Insula IV iv and its Relation to the Larger City (unpublished MA Thesis). University of Leiden. Leiden.
} 
con successo pioneristici metodi di analisi spaziale formale (space syntax) all'indagine sul contesto urbano di Ostia. Questo approccio si é rivelato come una valida ed adeguata strategia di ricerca per acquisire una più profonda comprensione di questo particolare quartiere all'interno del suo contesto urbano. Lo studio è stato in grado di dimostrare che il "quartiere Insula" (IV iv), nonostante sia composto da 14 edifici individuali, costituisse essenzialmente un insieme collettivo (fig. 2). La struttura spaziale del blocco cittadino era mediata da spazi comuni accessibili sia per i residenti che per i visitatori. Questi spazi comuni consistevano in una serie di cortili interconnessi tra loro che creavano una varietà di percorsi di circolazione, alcuni dei quali avevano una funzione specifica (raggiungere un'officina di lavoro quanto uno spazio commerciale), mentre altri rappresentavano l'occasione ideale per articolare relazioni sociali formali ed informali all'interno del quartiere. Riguardo la qualità dell'Insula dal punto di vista dello spazio vissuto, in molti casi è stato possibile stabilire che tali spazi erano progettati ed utilizzati proprio per promuovere gli incontri tra $\mathrm{i}$ residenti e tra $\mathrm{i}$ residenti ed i visitatori. I cortili e gli spazi di passaggio interni all'Insula sembrano aver generato dei rapporti di reciprocità, privilegiando l'integrazione piuttosto che la segregazione e l'esclusione (fig. 3 e 4). Le corti interne forniscono protezione internamente ma anche apertura verso l'esterno. La capacità dell'Insula di generare incontri sociali l'avrebbe resa un ambiente urbano amichevole e sicuro e avrebbe quindi conferito qualità allo spazio urbano, elemento molto apprezzato non solo nell'Ostia Romana di II ed inizio III suc, d.C., ma altamente rilevante anche nelle città di oggi.

Proprio la capacità di integrazione di questo blocco cittadino sembra esserne la chiave per il suo lungo periodo di occupazione (dal I sec. a.C. al V sec. d.C.). La sua organica struttura spaziale (fig. 3) pare averne impedito la frammentazione in unità abitative di lusso fortemente indipendenti, destino comune delle Insulea romane in quel particolare periodo storico (Becatti 1948). Nel epoca Tardo Antica infatti un gran numero di Insulae ostiensi ha assistito alla parziale conversione di singoli edifici in domus di lusso. L'inserimento di abitazioni di lusso all'interno dell'esistente tessuto urbano rappresenta un fenomeno molto simile agli attuali processi di gentrification che interessano le città moderne (Vedi per esempio Lees, Slater e Wyly 2008).

Nel complesso il blocco IV ii presenta chiaramente le caratteristiche di un quartiere. Questo infatti è dotato di quegli spazi che contribuiscono in maniera decisiva alla vitalità dell' unità urbana: cortili interni condivisi, spazi di passaggio comuni, portici protettivi e persorsi di spostamento che si intersecano (fig.3). Inoltre la natura autosufficiente del blocco suggerisce che questo possa aver funzionato come un coerente quartiere a sé stante, oltre ad essere parte di una più larga unità urbana, nella fattispecie una delle cinque regioni nelle quali la città antica era suddivisa $(C I L$ XIV 353, Vedi Bakker 1994: 197).

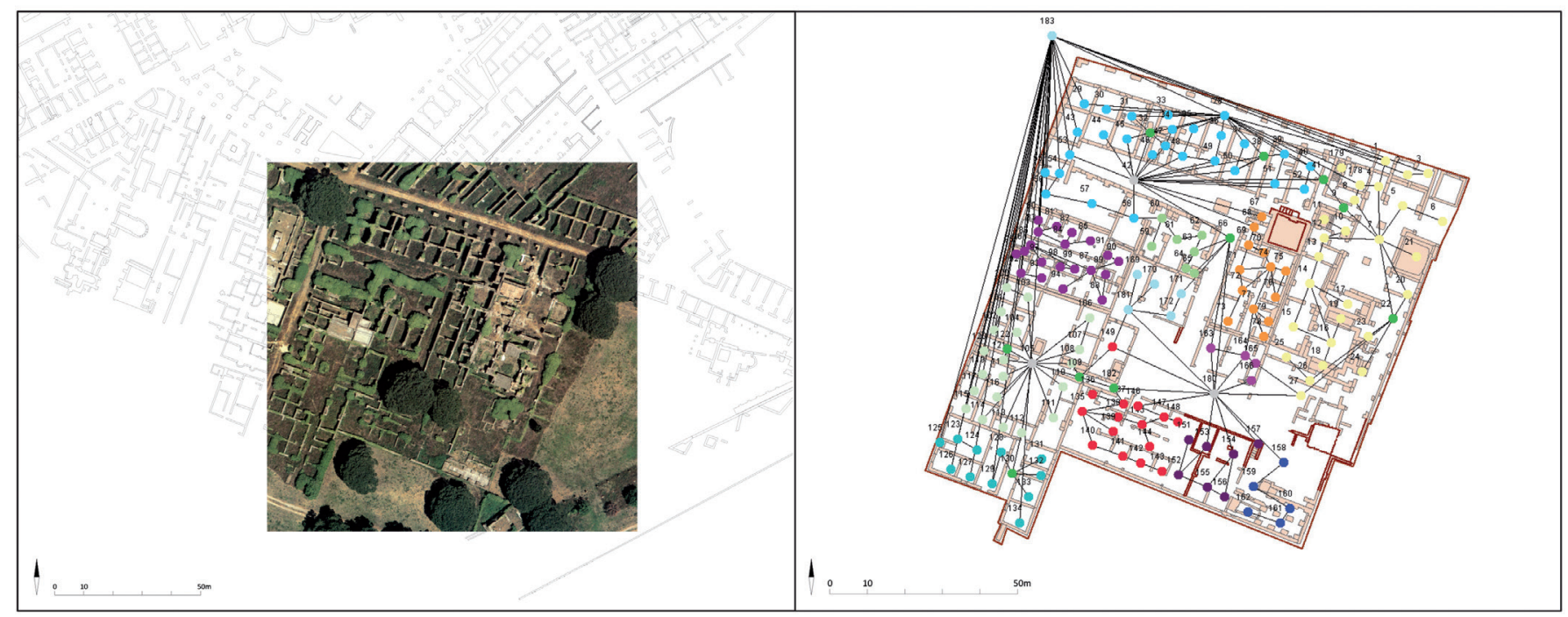

Figura 2. Insula IV ii, il grafico topologico della configurazione totale comprende 183 spazi individuali inclusi gli spazi di collegamento esterni (=183) (Fonte: Stöger 2011). 


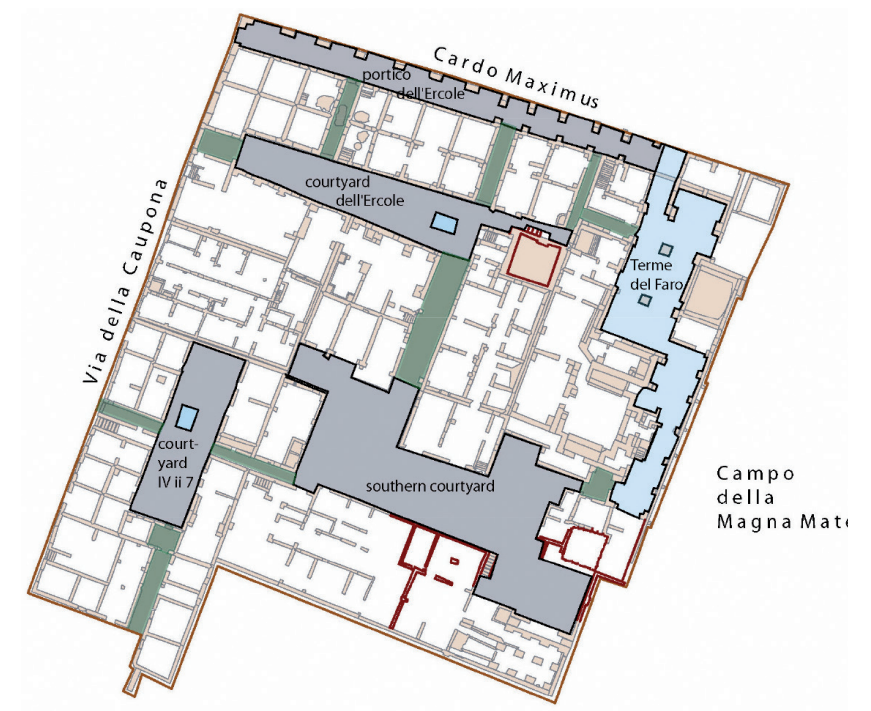

Figura 3. Blocco IV ii; la struttura dello spazio interno è caratterizzata da spazi collettivi interconnessi (corridoi in verde, cortili in grigio) compreso il complesso termale, le Terme del Faro IV ii 1 (celeste).

\begin{tabular}{|c|c|c|c|c|c|c|c|c|}
\hline Edificio & $\begin{array}{c}\text { Stanza/ } \\
\text { Funzione }\end{array}$ & No. & Profonditá & $\begin{array}{c}\text { RRA } \\
\text { (MRRA 0.937) }\end{array}$ & $\begin{array}{l}\text { Potenziale di } \\
\text { íntegrazione } \\
\text { globale }\end{array}$ & $\begin{array}{l}\text { Potenziale di } \\
\text { integrazione } \\
\text { locale }\end{array}$ & $\begin{array}{l}\text { Valori di } \\
\text { controllo }\end{array}$ & $\begin{array}{c}\text { Potenziale } \\
\text { disponibilitá di } \\
\text { presenza }\end{array}$ \\
\hline IV ii 1 & Entrata & 1 & 1.0 & 0.682 & Moderato & Basso & 0.432 & Mod/Basso \\
\hline IV ii 1 & Corridoio & 4 & 2.0 & 0.745 & Moderato & Basso & 0.793 & Mod/Basso \\
\hline IV ii 1 & Frigidarium & 7 & 3.0 & 0.735 & Moderato & Basso & 0.500 & Mod/Basso \\
\hline IV ii 1 & Passaggio & 9 & 4.0 & 0.838 & Moderato & Basso & 0.458 & Mod/Basso \\
\hline IV ii 1 & Passaggio & 22 & 4.0 & 0.784 & Moderato & Alto & 3.458 & Alto/Mod \\
\hline Comune & Cortile & 27 & 5.0 & 0.757 & Moderato & Basso & 0.759 & Mod/Basso \\
\hline IV ii 2 & Portico & 28 & 1.0 & 0.622 & Alto & Alto & 7.652 & Alto \\
\hline IV ii 3 & Corridoio & 32 & 2.0 & 0.658 & Moderato & Moderato & 1.371 & Moderato \\
\hline IV ii 3 & Passaggio & 38 & 2.0 & 0.659 & Moderato & Basso & 0.705 & Mod/Basso \\
\hline IV ii 3 & Passaggio & 41 & 3.0 & 0.694 & Moderato & Basso & 0.809 & Mod/Basso \\
\hline IV ii 3 & Cortile & 42 & 2.0 & 0.558 & Alto & Alto & 7.699 & Alto \\
\hline IV ii 3 & Passaggio & 47 & 3.0 & 0.742 & Moderato & Moderato & 1.009 & Moderato \\
\hline IV ii 3 & Entrata & 53 & 1.0 & 0.602 & Alto & Basso & 0.928 & Alto/Basso \\
\hline Comune & Passaggio & 66 & 3.0 & 0.617 & High & Moderate & 2.302 & Alto/Mod \\
\hline IV ii 6 & Corridoio & 86 & 1.0 & 0.733 & Moderate & High & 4.035 & Alto/Mod \\
\hline IV ii 7 & Cortile & 105 & 2.0 & 0.703 & Moderate & High & 9.416 & Alto/Mod \\
\hline IV ii 7 & Passaggio & 109 & 3.0 & 0.724 & Moderate & Moderate & 1.233 & Moderato \\
\hline IV ii 7 & Passaggio & 122 & 1.0 & 0.663 & Moderate & Low & 0.602 & Mod/Basso \\
\hline IV ii 8 & Passage & 130 & 3.0 & 0.973 & Moderate & High & 4.000 & High/mod \\
\hline Comune & Sud & 180 & 4.0 & 0.617 & High & High & 5.783 & High \\
\hline Comune & Fronte 14 & 181 & 5.0 & 0.783 & Moderato & High & 2.910 & Alto/Mod \\
\hline IV ii 13 & Passaggio & 182 & 4.0 & 0.699 & Moderato & Low & 0.660 & Mod/Basso \\
\hline Comune & $\begin{array}{l}\text { Spazio } \\
\text { esterno }\end{array}$ & 183 & 0.0 & 0.562 & Alto & Alto & 165.386 & Alto \\
\hline
\end{tabular}

Figura 4. (tabella) Gli spazi di spostamento e di incontro dell'Insula IV ii (segnati in grigio scuro): quelli direttamente connessi con lo spazio esterno sono segnati in grigio chiaro $(1,28,53,86,122$ e 130), a differenza dei cortili esclusivamente interni $(42,105$ e 180$)$ e degli spazi di trasporto esterni (183). 


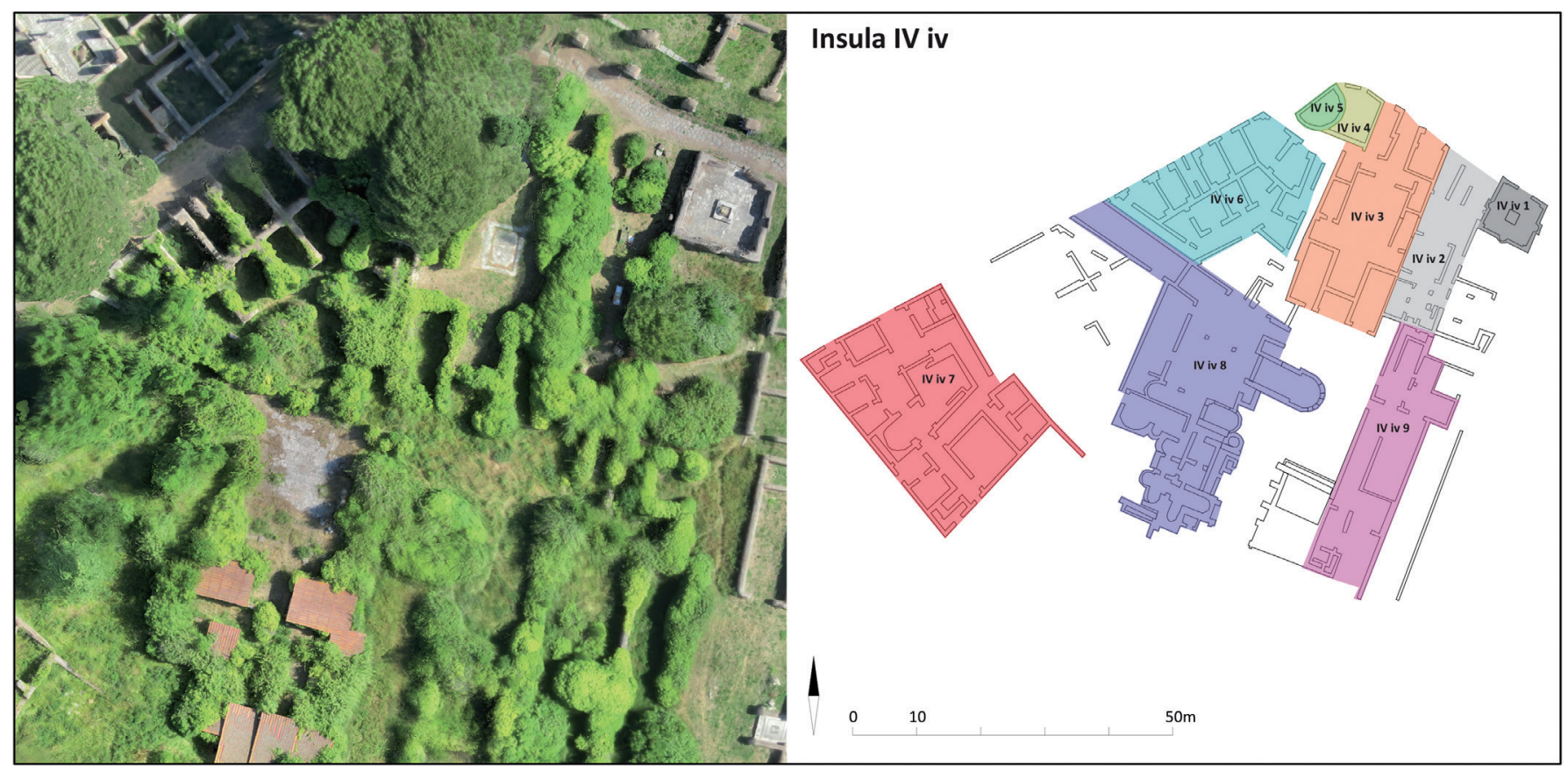

Figura 5. Blocco cittadino IV iv, un ortofoto a bassa altitudine mostra come l'insula sia invasa dalla vegetazione.

\section{BLOCCO CITTADINO IV IV. HA MAI EFFETTIVAMENTE FUNZIONATO COME UN QUARTIERE?}

Al fine di elaborare un'indagine più completa consideriamo una seconda Insula (IV iv) per uno studio comparativo (fig. 5). La sua notevole prossimità con il Forum e il suo lungo periodo di occupazione, che copre dal periodo Repubblicano a quello Tardo Antico, rendono l'Insula IV iv particolarmente interessante ai fini del nostro studio. Il blocco comprendeva nove edifici individuali, alcuni dei quali riflettevano i principi tradizionali della città romana ed altri la sua vivace energia di rinnovamento. Gli edifici in questione includono quattro abitazioni residenziali (Domus IV iv 2, 3, 7 e 9), un blocco residenziale (Caseggiato IV iv 6), un complesso termale (Terme bizantine IV iv 8), due fontane (Ninfei IV iv 1 e 5), ed una latrina pubblica (Forica IV iv 4). Questo specifico quartiere urbano (IV iv) garantisce un'abitazione ai suoi residenti per ben più di sette secoli.

Situata all'angolo dove il Cardo Massimo e la Via del Tempio Rotondo si intersecano ed incontrano il $\mathrm{Fo}$ rum, l'Insula IV iv godeva di una posizione dominante e centrale nell'ambito cittadino. Il blocco é delimitato da strade sui quattro lati: il suo confine a nord è costituito dal Cardo Massimo di Ostia, mentre a sud si estende fino alla Via di Iside e a nord-ovest fino a Via del Tempio Rotondo; sul lato sud-est invece uno stretto vicolo la separa dall'Insula IV iii (fig 6).

Contrariamente all'Insula IV ii precedentemente analizzata, la cui struttura era basata sulla comunione degli spazi, l'Insula IV iv è caratterizzata da edifici individuali che non condividono spazi comuni collettivi, ma piuttosto erano proiettati verso l'esterno, tutti con accessi diretti verso lo spazio pubblico ad eccezione delle terme tardo antiche (IV iv 8) che si sono annidate al centro dell'Insula durante l'ultimo periodo di insediamento (V sec. d.C.). Durante il loro lungo periodo di occupazione i nove edifici hanno subito numerose trasformazioni in risposta ai cambiamenti nell'economia della città e al modo in cui i propri abitanti concepivano ed interagivano con lo spazio. Il blocco vanta la presenza di due fontane (nymphaea) lungo il prestigioso Cardo Massimo, una delle strade maggiori che conduceva dal centro della città alla porta meridionale. Se da una parte queste fontane rappresentavano dei potenziali poli per il quartiere, risulta evidente che fossero rivolte allo spazio pubblico, finalizzate appunto ad attrarre l'attenzione dei visitatori e dei passanti. Nel complesso l'Insula non sembra particolarmente interessata a creare un'identità collettiva attraverso la condivisione degli spazi, il che porta a chiedersi se abbia mai effettivamente funzionato come un vero e proprio quartiere o se abbia invece fatto parte di un'unità urbana più estesa dei suoi confini. 


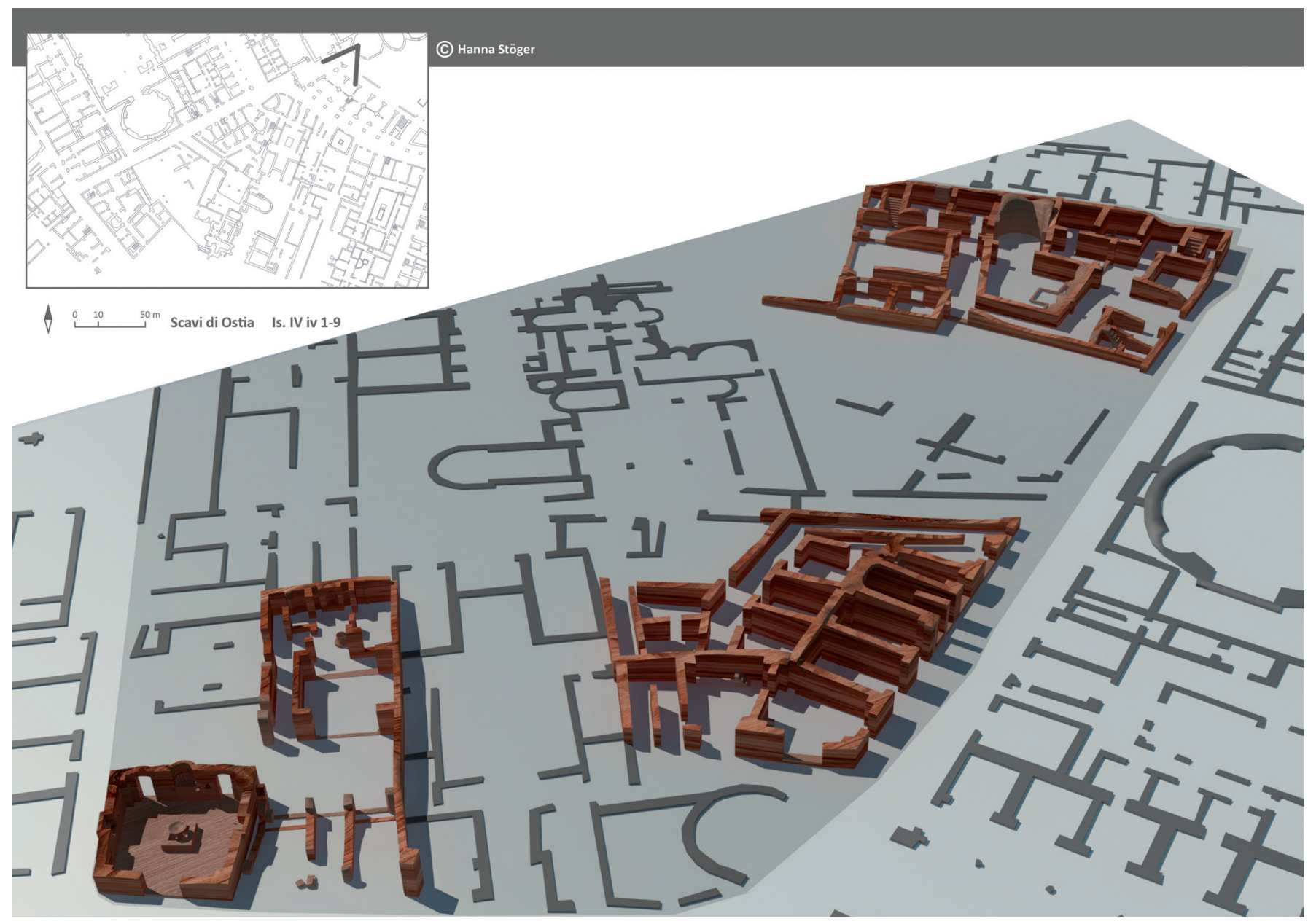

Figura 6. Visualizzazione 3D del blocco IV; nell'ambito di questa ricerca si sta portando avanti la ricostruzione 3D dei dati archeologigi raccolti durante questi anni. Ad oggi è stato copletato il modello 3D di 3 dei 9 edifici pervenutici non solo a scopo di visualizzazione ma anche come prezioso e versatile contenitore dati.

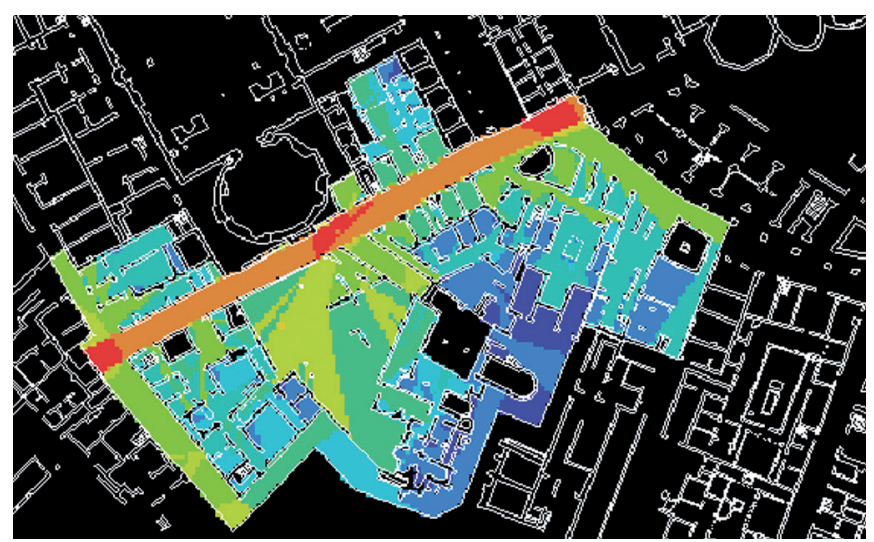

Figura 7. La Via del Tempio Rotondo fa parte di un piú ampio quartiere urbano; VGA dell'integrazione visuale lungo la via del Tempio Rotondo. I valori d'integrazione piú elevati si riscontrano sulla strada, al centro di questa dove la visibilitá converge e nel punto di intersezione con altre strade (Integrazione $\mathrm{HH}$ : Media = 340,844; Minima = 147,015; Massima $=$ 594,093).

Troviamo varie indicazioni, soprattutto lungo la Via del Tempio Rotondo, a supporto di quest'ultima ipotesi. Gli edifici che si affacciano su entrambi i lati di questa via presentano infatti una notevole corrispondenza e risalgono allo stesso periodo di sviluppo in età severiana (primo quarto del III sec. d.C.), suggerendo la presenza di un unità coesa (fig. 7). All'estremo settentrionale della strada, ad esempio, la facciata del blocco residenziale (Caseggiato IV iv 6), sembra interagire con quella della Domus del Tempio Rotondo (I IX 2-3) dal lato opposto. Questi edifici sono direttamente l'uno di fronte all'altro e la corrispondenza degli accessi ne permetteva il reciproco controllo visivo (fig. 8). Anche sul lato sud della Via del Tempio Rotondo, prima che questa si intersechi con la Via di Iside, troviamo due edifici con fronti stradali corrispondenti. La Domus su Via del Tempio Rotondo (IV iv 7), posizionata all'angolo sud-ovest dell'Insula, costituisce di fatto un parallelo dell'edificio dall'altra parte della strada (Edificio I x 4, edificio corporativo che ospitava il Tempio Collegiale ed il Mitreo di Fructosus). Gli edifici sono dimensionalmente molto simili ed hanno entrambi i fronti stradali chiusi lungo il tratto sud della Via del Tempio Rotondo, contribuendo a creare un'impressione visiva omogenea ed 


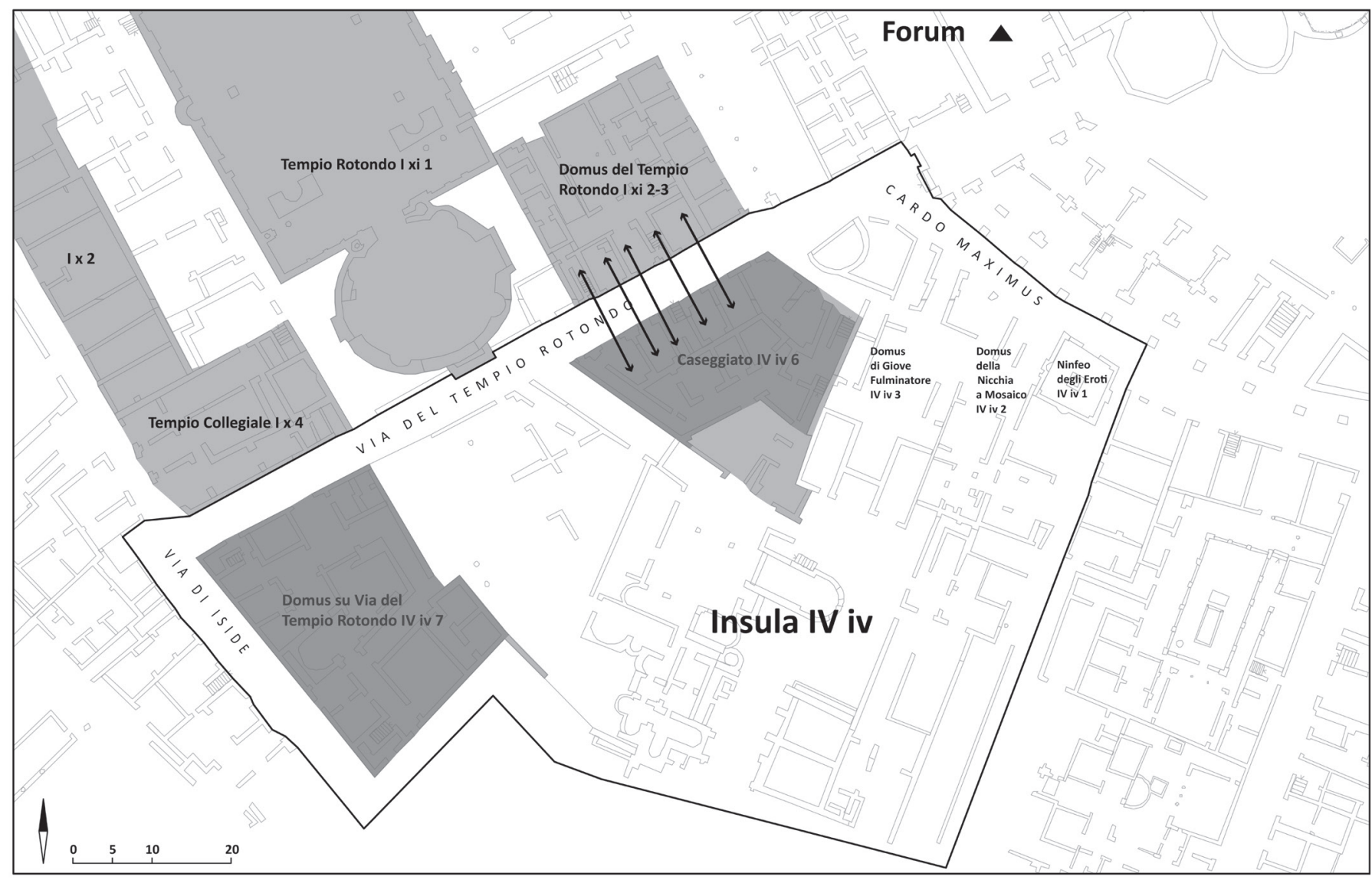

Figura 8. Blocco-facciata ('face-block') lungo la Via del Tempio Rotondo che comprende il Caseggiato IV iv e la Domus del Tempio Rotondo (I xi 2-3). Da notare l'interazione tra i fronti stradali e il controllo visuale reciproco fra questi edifici. Le aree oggetto del progetto di rinnovamento urbano di Etá Severiana sono segnate in grigio.

unitaria. Gli edifici sopraelencati sembrano tutti far parte di un progetto di rinnovamento urbano comune, connesso alla costruzione del monumentale Tempio Rotondo (I xi 1). Questo si caratterizza come l'ultimo grande tempio costruito ad Ostia prima del graduale declino subito dalla città a partire dal III sec., in corrispondenza della progressiva trasformazione in città cristiana, con tanto di basilica e sede vescovile, definitivamente abbandonata tra VIII e IX sec. d.C.

Volendo dunque parlare di quartiere, nel caso dell' Insula IV iv, è necessario estendere la sua portata sociale oltre i suoi confini fisici e concettualizzare un'unità urbana più ampia che potrebbe aver incluso l'intero blocco IV iv, o solo la sua parte settentrionale lungo la Via del Tempio Rotondo. Lungo questa strada infatti sembrano essere presenti molte delle caratteristiche che definiscono un quartiere, a partire dalla già citata interazione architettonica tra le facciate degli edifici che vi si affacciano (fig. 8). D'altra parte però, il blocco IV iv di per sé non può essere inequivocabilmente qualificato come quartiere; questo infatti è sprovvisto di quegli spazi comuni che contribuiscono a creare un senso di comunità. Inoltre, $\mathrm{i}$ singoli edifici sembrano aver conservato la loro indipendenza spaziale nell'arco della maggior parte del tempo che sono stati utilizzati. Solo successivamente, in una più tarda fase (tra fine IV ed inizio $\mathrm{V}$ sec.) un complesso termale venne inserito al centro dell'Insula. Le terme potrebbero aver assunto la funzione di fulcro della vita sociale e quindi contribuito alla creazione di un senso di quartiere all'interno del blocco IV iv.

\section{UN'ANALISI SPACE SYNTAX SUGLI EDIFICI LUNGO LA VIA DEL TEMPIO ROTONDO}

Uno sguardo più da vicino ad uno degli edifici prospicienti la Via del Tempio Rotondo aiuta a comprendere più a fondo la relazione tra $\mathrm{i}$ vari edifici ed il loro assetto locale. L'analisi del blocco residenziale Caseggiato IV iv 6 permette di individuare diversi fattori che potrebbero averne influenzato la funzione o addirittura 


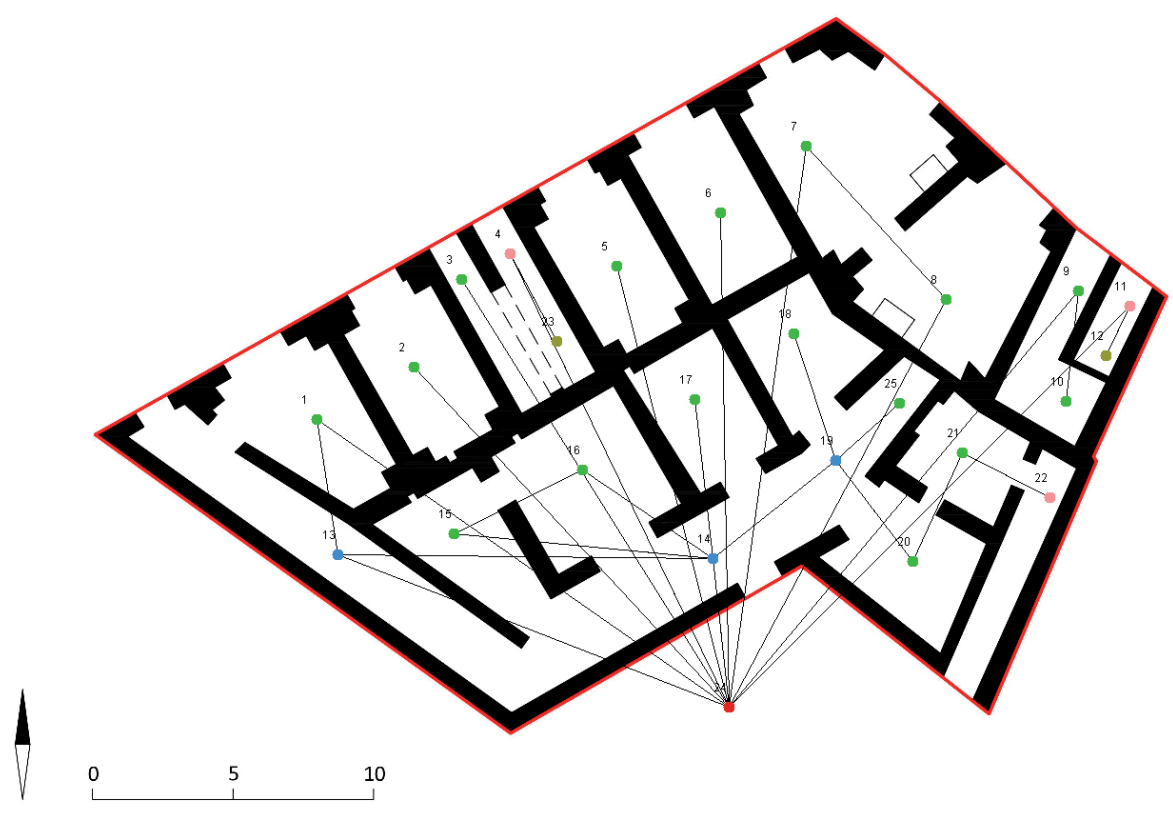

Figura 9. Caseggiato (IV iv 6), grafico topologico (prodotto utilizzando il programma JASS, KTH Stockholm). plasmato la forma. L'edificio è situato in corrispondenza dell'intersezione tra la Via del Tempio Rotondo ed il tratto meridionale del Cardo Massimo ed il suo schema trapezoidale rivela un uso dello spazio che si è adattato agli edifici preesistenti. Nella sua parte nord questo risponde architettonicamente alla forma semicircolare del ninfeo (IV iv 5) arretrando verso l'interno. Il suo lato sud è delimitato attualmente dall'area delle terme (Terme bizantine IV iv 8), mentre nel periodo risalente alla sua costruzione era definito dalle strutture preesistenti ad esse. Ad est invece è fiancheggiato dalla Domus di Giove Fulminatore (IV iv 3), mantenendo però una chiara divisione di proprietà tra gli edifici. E' opportuno puntualizzare infatti che non vi sono pareti divisorie condivise tra il Caseggiato IV iv 6 e la domus, con il muro divisorio orientale del primo allineato parallelamente a quello occidentale della seconda. Il Caseggiato, insieme ad altri edifici lungo la Via del Tempio Rotondo, tutti risalenti al periodo severiano, (all'incirca il primo quarto del III sec. d.C.), rientrava nell'ottica del già citato organico programma di edificazione associato alla costruzione del monumentale Tempio Rotondo.

Il piano terra dell'edificio (IV iv 6 ) è ben conservato, con le mura sopravvissute, alte dai tre o quattro metri e tre corpi scala che conducevano ai piani superiori non pervenutici. La distribuzione interna dello spazio, che comprende 20 stanze con corridoi e scale, denota una netta divisione in due compartimenti ben distinti. Mentre tutte le stanze del lato nord si aprono verso la strada, ognunua con il proprio accesso diretto $(1,2,5,6,7,8,9$ ed i vani scale 3, 4 e 11), tutte le stanze meridionali (15, $16,17,18,20$ e 25$)$ sono raggiungibili solo attraverso una serie di corridoi (13, 14 e 19) (fig. 9).

Una così netta distinzione indica chiaramente che le stanze a sud erano destinate ad uso residenziale, mentre tutte le stanze a nord avevano una funzione commerciale. Il J-graph (grafico giustificato) (fig. 10) illustra chiaramente questa divisione in settore commerciale semi-pubblico da una parte e settore residenziale privato dall'altra. Questa compartimentazione sembra essere stata motivata anche dalla particolare posizione dell'edificio nel contesto cittadino. La vicinanza al Forum e la sua posizione ad angolo infatti lo rendevano allo stesso tempo un luogo privilegiato per gli scambi commerciali ed un prestigioso indirizzo presso il quale risiedere. La parte residenziale sul lato sud, protetta ed appartata, era accessibile dalla strada soltanto per mezzo di un corridoio mentre dall'interno dell'Insula stessa attraverso una corte interna. Le stanze lungo il fronte stradale $(1,2,5$ e 6$)$ sono caratterizzate da dimensioni pressoché equivalenti ad eccezione delle stanze 7 e 8, collegate tra loro e di dimensioni maggiori. I risultati preliminari delle operazioni di scavo tuttora in corso suggeriscono che queste due stanze ospitassero attività di laboratorio. Queste rappresentavano di fatto locali perfetti per delle officine combinate a negozi, un tipo d'uso funzionale supportato dall'attrattività commerciale della posizione in prossimità del Forum e del Cardo. Questa vicinanza al centro ne influenza anche la configurazione spaziale, che, ad esempio, ne nascondeva la parte produttiva a chi vi si avvicinava dal foro. In particolare la 


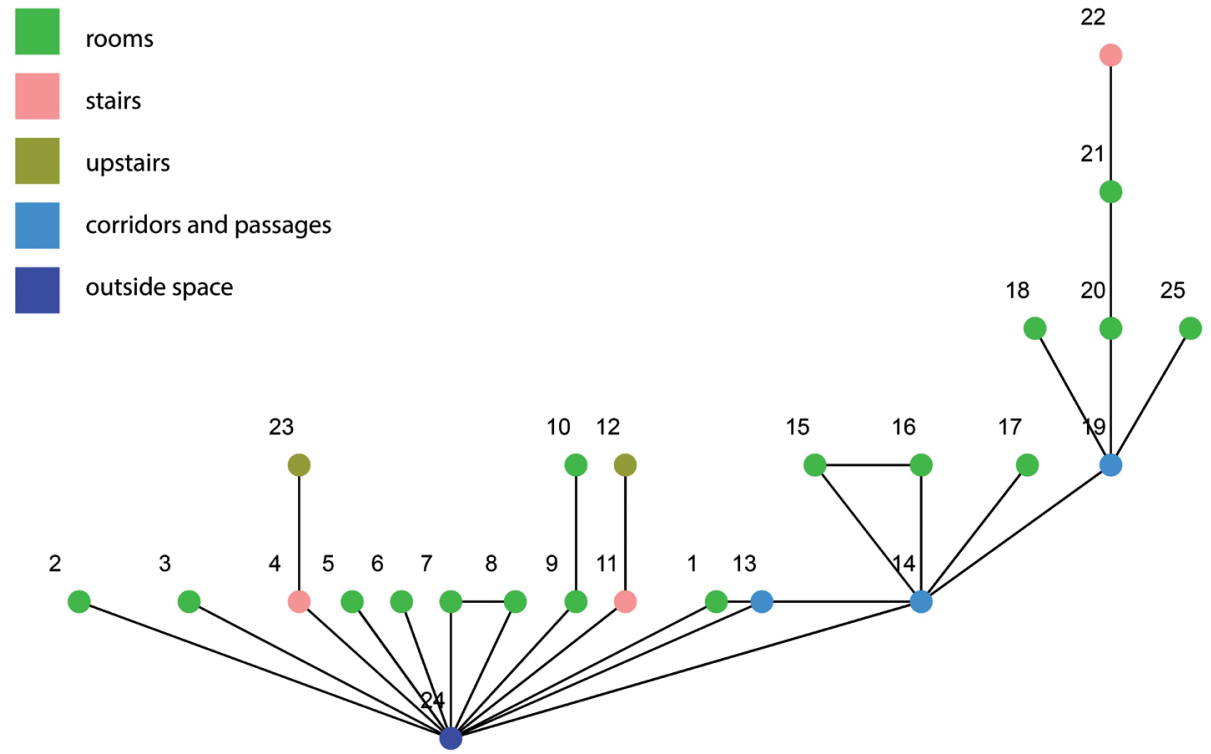

Figura 10. Caseggiato IV iv $6 \mathrm{j}$-graph: tutti gli spazi commerciali lungo il fronte stradale $(1,2,5,6,7$ e 8$)$ sono direttamente connessi allo spazio pubblico; i locali residenziali $(14,15,16,17,18,19$ e 25) al lato sud dell'edificio sono invece chiusi verso I'interno garantendo una certa riservatezza nonostante la prossimitá del centro cittadino. 13,14 e 19 sono dei corridoi di collegamento che generano movimento ( $\mathrm{j}$-graph prodotto utilizzando il programma JASS). stanza d'angolo (7), grazie alle sue due apreture, appare ben integrata da punto di vista visuale e sembra aver fatto buon uso della sua speciale posizione, come risulta chiaro dal VGA di figura 7. Da notare come la porta che si apre sulla Via del Tempio Rotondo sia relativamente stretta $(1.11 \mathrm{~m})$, mentre quella verso il ninfeo è decisamente più larga $(2.53 \mathrm{~m})$. Questa differenza potrebbe essere dettata da esigenze strutturali, ma è anche possibile ipotizzare che la divisione funzionale possa aver definito la dimensione delle aperture delle stanze 7 e 8 .

Il j-graph (fig. 10) rivela inoltre una poco profonda struttura ramificata con ben 12 spazi, più della metà delle stanze dell'edificio, direttamente collegati allo

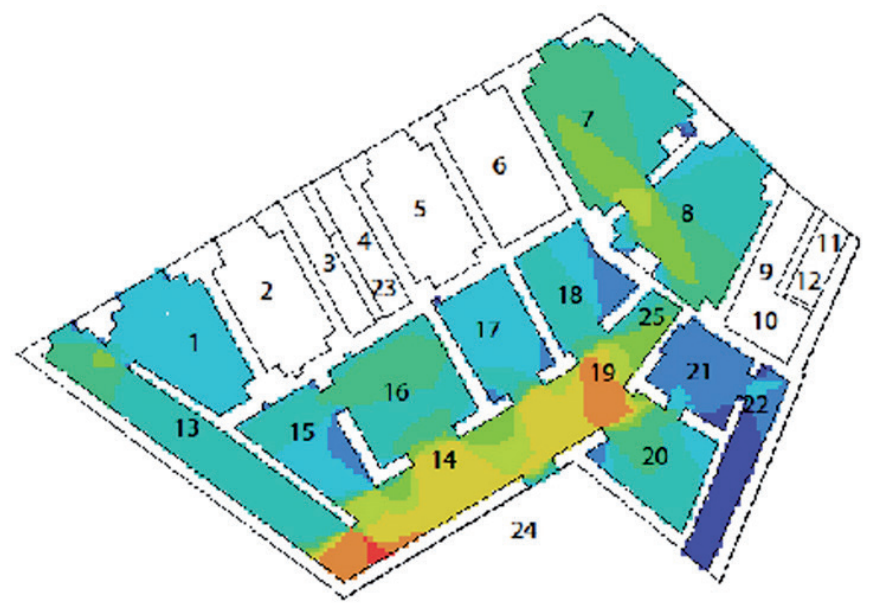

Figura 11. Caseggiato IV iv 6, VGA degli spazi interconnessi al piano terra dell'edificio. Gli spazi meglio integrati visivamente sono gli spazi di passaggio ed i corridoi (13, 14 e 19) che conducono agli alloggi privati nella parte meridionale dell'edificio. L'analisi degli accessi (Figura 9) ed il VGA si confermano a vicenda, individuando gli spazi di passaggio (14 e 19) come le zone meglio integrate dell'edificio; (VGA generato utilizzando Depthmap, UCL). spazio pubblico. La struttura spaziale dell'edificio ha una profondità di 5 'stepdepth' dall'esterno, mentre tutti gli spazi che suggeriscono una destinazione d'uso residenziale sono profondi circa 2 passi di profonditá. L'organizzazione spaziale dell'edificio non promuove la circolazione esterna, mentre favorisce gli spostamenti lineari tra le stanze all'interno.

Per quanto riguarda le stanze per le quali è stato suggerito un uso residenziale, i corridoi attraverso i quali vi si accede ne costituiscono anche un elemento di mediazione (fig. 11). La disposizione delle stanze 15, 16,17 e 18 , con il corridoio centrale 14 , rimanda alla cosidetta abitazione medianum, una tipologia abitativa in cui il corridoio centrale sostituisce il tradizionale cortile della tipologia domus delle case romane (Vedi DeLaine 2004). Queste particolari abitazioni erano molto diffuse ad Ostia tra i cittadini più abbienti ed influenti, ulteriore conferma di come l'eccellente posizione dell'edificio lo rendeva un attrente luogo di residenza.

\section{Il quartiere e le infrastrutture urbane}

Se da una parte i blocchi cittadini ostiensi sono delle unità spaziali autosufficienti, dall'altra sono anche delle componenti della città, collegate attraverso l'apparato della rete stradale ad edifici pubblici, infrastrutture e altri servizi condivisi. Strumenti della space syntax sono stati utilizzati anche per l'analisi dell'estesa rete stradale di Ostia, che comprende 467 strade, incluse quelle rilevate nella parte non scavata attraverso prospezioni geofisiche (cf. Martin e Heinzelmann 2000). L'analisi assiale (Axial 


\begin{tabular}{|c|c|c|}
\hline Valore & $\begin{array}{c}\text { Integrazione (HH) } \\
\text { Tutte le strade } \\
\text { (n-467) }\end{array}$ & $\begin{array}{c}\text { Selezione } \\
\text { Via del Tempio } \\
\text { Rotondo }\end{array}$ \\
\hline Media & 1.01881 & 1.38441 \\
\hline Minimo & 0.532099 & 1.38441 \\
\hline Massimo & 1.84646 & 1.38441 \\
\hline Standard Div. & 0.227051 & 0 \\
\hline Conto & 467 & 1 \\
\hline
\end{tabular}

Figura 12. (tabella) Analisi assiale: Valori di Integrazione (HH) per la rete viaria di Ostia, selezione: Via del Tempio Rotondo.

Analysis), in particolare, ha individuato degli elevati valori di integrazione per la Via del Tempio Rotondo (fig. 12). Questo risultato non è affatto sorprendente in virtù della sua posizione centrale e del fatto che, nonostante la sua lunghezza relativamente ridotta (di soli $100 \mathrm{~m}$ ), si interseca con ben altre nove strade. La Via del Tempio Rotondo è posta infatti nel punto di convergenza tra le vie che connettevano il Forum al resto della città (il Decumano Massimo e il Cardo Massimo) e i collegamenti della città a scala territoriale (la Via Ostiense che connette Ostia con Roma; la Via della Foce, che conduce alla foce del fiume Tevere e la Via Laurentina che collega Ostia con le zone rurali a sud-est della città) (fig. 13). I luoghi dove queste scale locali e regionali si intersecano sono generalmente dotati di un grande potenziale nella fabbrica urbana, assumendo spesso una funzione di centrale importanza. Queste sono le strade principali dove il traffico regionale e i residenti locali si incontrano ed interagiscono, formando così delle arene ideali per le attività politiche, religiose, amministrative e commerciali. Considerando la concentrazione di fattori spaziali favorevoli che convergono in questo punto, non sorprende che tutti gli ultimi progetti di rinnovamento urbano a larga scala si sono concentrati in questa specifica area della città, focalizzandosi in particolare sulla Via del Tempio Rotondo.

\section{CONCLUSIONI}

Questo studio sui quartieri ostiensi consente di esaminare attentamente le proprietà spaziali di due blocchi della città romana (Insulae IV ii e iv). L'organizzazione dello spazio fisico del blocco IV ii, attraverso la condivisione di cortili, passaggi e portici, ne suggerisce un uso comune e collettivo che ne può aver facilitato lo sviluppo come unità quartiere. All'interno del blocco IV ii, i cui confini sono definiti dalla struttura a maglie della rete stradale, sembra essere stata incoraggiata la creazione di spazi di attiva collettività. Ricorrenti tipi di spostamenti od incontri possono aver rinforzato la consapevolezza dei residenti e la loro coesione socile, ma hanno anche stimolato e promosso allo stesso tempo la compresenza di residenti e visitatori. Gli approfondimenti sulle dinamiche spaziali del blocco ottenuti grazie all'utilizzo della Space Syntax hanno portato a supporre che la sua struttura spaziale abbia contribuito in maniera decisiva alla formazione del quartiere e ne abbia soprattutto sostenuto la struttura collettiva lungo un periodo di tempo di quasi cinque secoli (dal I al V sec. d.C.). Al contrario, lo studio comparativo del blocco IV iv ha rivelato una differente organizzazione di quartiere. Questo infatti si è sviluppato e focalizzato in particolare lungo una strada, la Via del Tempio Rotondo. A sostegno di questa ipotesi possono essere identificati, su entrambi i fronti stradali di questa direttrice, numerosi fattori spaziali che hanno contribuito alla creazione di una vita di quartiere e di

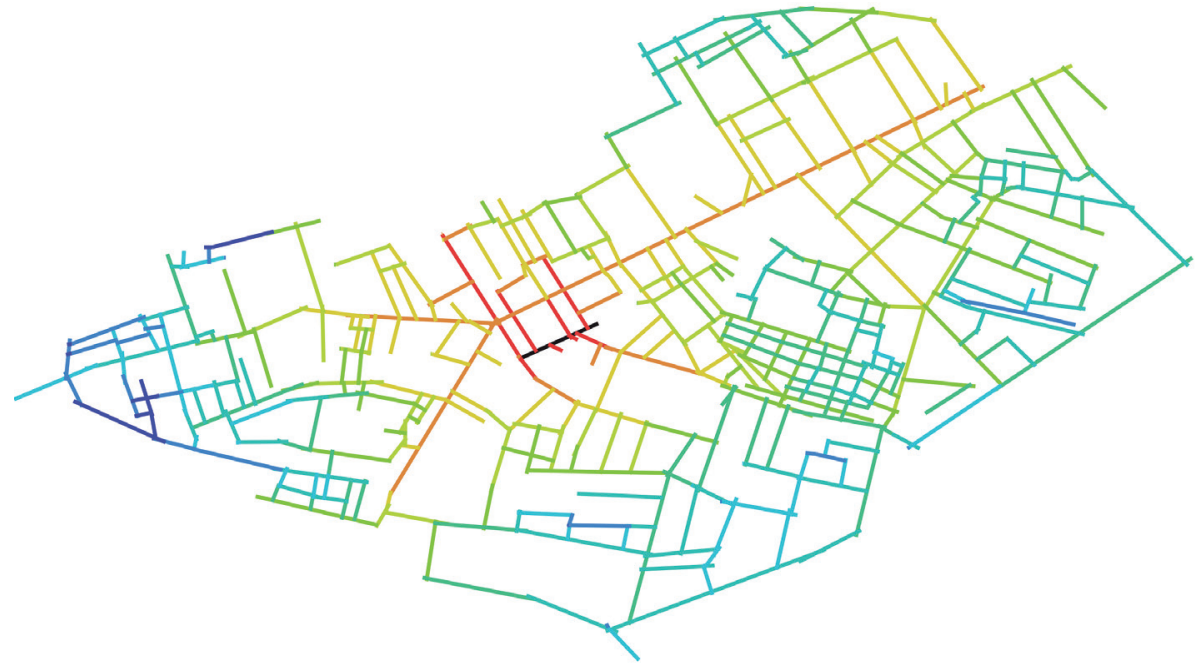

Figura 13. L'estesa rete stradale ostiense (ben 467 strade), profonditá angolare calcolata dalla Via del Tempio Rotondo (indicata in nero). Il grafico evidenzia le strade che erano agevolmente accessibili dalla Via del Tempio Rotondo e che con essa formavano il contesto stradale locale (grafico prodotto utilizzando Depthmap, UCL). 
una vera e propria comunità urbana. Ciò estende la portata sociale del blocco IV iv al di là dei suoi confini fisici, includendo possibilmente anche aree al di fuori del blocco stesso. L'integrazione e la strategica posizione della Via del Tempio Rotondo all'interno della rete stradale ostiense gli permettevano inoltre di trarre beneficio sia dal vicino centro cittadino sia dai percorsi d'accesso che portavano le attività regionali all'interno della città. Questa strada sembra aver assunto un ruolo di centrale importanza nell'economia della città, in grado di attrarre investimenti per il rinnovamento urbano, nonostante fosse già cominciata la graduale fase di trasformazione demografica ed architettonica che prefigurava il suo lento declino.

La prospettiva della Space Syntax, applicata al caso studio di due quartieri di Ostia Antica, ci ha permesso di approfondire la flessibile struttura urbana delle antiche città romane. I metodi sintattici offrono un approccio non invasivo all'investigazione dell'archeologia urbana ed una preziosa quantità di dati spaziali in grado di rivelare suggestivi tratti della natura delle comunità urbane che vivevano tra $\mathrm{i}$ blocchi cittadini di Ostia Antica.

\section{RIFERIMENTI BIBLIOGRAFICI}

Allison, P. (2004): Pompeii Households: Analysis of the Material Culture. Cotsen Institute of Archaeology, UCLA. Los Angeles.

Arnauld, M. C., Manzanilla, L. R. y Smith, M. E. (2012): The Neighbourhood as a Social and Spatial Unit in Mesoamerican Society. University of Arizona Press. Tucson.

Bakker, J. T. (1994): Living and Working with the Gods: Studies of Evidence for Private Religion and its Material Environment in Ostia $(100 \mathrm{BC}-500$ AD). J. Gieben. Amsterdam.

Becatti, G. (1948): »Case ostiensi del tardo impero«. Bollettino di Archeologia. 33 (102.128):197-224.

Bermejo, J. (2014): Arqueología Biopolitica. La sintaxis espacial de la arquitectura doméstica romana en la Meseta oriental. Ediciones La Ergástula. Madrid.

Bert Lott, J. (2004): The Neighbourhoods of Augustan Rome. Cambridge University Press. Cambridge.

Boersma, J. S. (1985): Amoenissima Civitas, Block V.ii at Ostia: Description and Analysis of its Visible Remains. Van Gorcum. Assen.

Bowman, A. y Wilson, A. (Ed.) (2011): Settlement, Urbanisation, and Population. Oxford University Press. Oxford and New York.

Dalton, N. S. (2006): 'Configuration and neighbourhood: Is place measurable?'. En Hoelscher, C., Conroy Dalton, R. and Turner, A. (Ed.): Space Syntax and Spatial Cognition, Proceedings of the Workshop held in Bremen, 24th September 2006. University of Bremen. Bremen: 53-65

DeLaine, J. (2004): »Designing for a market: 'medianum' apartments at Ostia«. Journal of Roman Archaeology. 17: 147-176.

Düring, B. S. (2006): Constructing Communities: Clustered Neighbourhood Settlements of the Central Anatolian Neolithic ca 8500-5500 BC. Nederlands Instituut voor het Nabije Oosten. Leiden.

Gans, H. J. (1962): The Urban Villagers. Group and Class in the Liefe of Italian-Americans. The Free Press of Glencoe. New York.
Gering, A. (2002): »Die case a giardino als unerfüllter Architektentraum: Planung und gewandelte Nutzung einer Luxuswohnanlage im antiken Ostia«. Mitteilungen des Deutschen Archäologischen Instituts Römische Abteilung. 109: 109-140.

Grahame, M. (2000): Reading Space: Social Interaction and Identity in the Houses of Roman Pompeii: A syntactical approach to the analysis and interpretation of built space. BAR International Series 886. Archaeopress. Oxford.

Guidobaldi, F. (1995): »Una Domus Tardoantica Inedita di Ostia ed I suoi Pavimenti«. Atti del II Colloquio dell'Associazione italiana per lo studio e la conservazione del mosaico, AISCOM. Colloquio Roma 1994: 525-535.

Hillier, B. (1996): Space is the Machine: A Configurational Theory of Architecture. Cambridge University Press. Cambridge.

Hillier, B. (2008): »Space and spatiality: What the built environment needs form social theory«. Building Research and Information. 36 (3): 216-230.

Hillier, B. y Hanson, J. (1984): The Social Logic of Space. Cambridge University Press. Cambridge.

Hillier, B. y Vaughan, L. (2007): »The city as one thing«. Progress in Planning. Vol. 67 (3): 205-230.

Jones, R. y Robinson, D. J. (2007): »Intensification, heterogeneity and power in the development of Insula VI, 1«. En J. J. Dobbins y P. W. Foss (Ed.): The World of Pompeii. Routledge. London and New York: 119-128

Kaiser, A. (2000): The Urban Dialogue: An Analysis of the Use of Space in the Roman City of Empuries, Spain, BAR International Series 901. Archaeopress. Oxford.

Kaiser, A. (2011): Roman Urban Street Networks. Routledge. New York and London.

Laurence, R. (2007 [1994]): Roman Pompeii: Space and Society. Routledge. London and New York.

Laurence, R., Esmonde Cleary, S. y Sears, G. (2011): The City in the Roman West, c. 250 BC - c. AD 250. Cambridge University Press. Cambridge and New York.

Lees, L., Slater, T., and Wyly, E. K. (2008): Gentrification. Routledge. London and New York.

Lorenzatti, S. (1998): »La Domus di Giove Fulminatore«. Bolletino di Archeologia dal ministero per I beni culturali e ambientali: 49-50, 79-98.

Martin, A. y Heinzelmann, M. (2000): »The joint AAR-DAI Research Project at Ostia: 1998 and 1999 seasons «. Memoirs of the American Academy in Rome. 45: 277-283.

Medri, M. e Di Cola, V. (2013): Ostia V Le Terme del Nuotatore. L'Erma di Bretschneider.Rome.

Moulaert, F., Martinelli, F., Swyngedouw, E. y Gonzalez, S. (2013 [2010]): Can Neighbourhoods Save the City? Routledge, London and New York.

Paliou, E., Lieberwirth, U. y Polla, S. (Ed.) (2014): Spatial Analysis and Social Spaces: Interdisciplinary Approaches to the Interpretation of Prehistoric and Historic Built Environments,. De Gruyter, Berlin and Boston: 297-316

Sampson, R. J. Morenoff, J. D., y Gannon-Rowley, T. (2001): »Assessing neighbourhood effects: Social processes and new directions in research «. Annual Review of Sociology. 28: 443-478.

Scott, M. (2012): Space and Society in the Greek and Roman Worlds, Key Themes in Ancient History. Cambridge University Press. Cambridge and New York.

Smith, M. E. (2010a): »The archaeological study of neighborhoods and districts in ancient cities«. Journal of Anthropological Archaeology. 29 (2):137-154.

Smith, M.E. (2010b): »Sprawl, squatters and sustainable cities: Can archaeological data shed light on modern urban issues?«. Cambridge Archaeological Journal. 20 (2): 229-253.

Stöger, H. (2011): Rethinking Ostia: A Spatial Enquiry into the Urban Society of Rome's Imperial Port-Town, Archaeological Studies Leiden University 24. Leiden University Press. Leiden.

Stöger, H. (2014): »The spatial signature of an Insula neighbourhood of Roman Ostia«. En Paliou, E., Lieberwirth, U. y Polla, S. (Ed.): Spatial Analysis and Social Spaces: Interdisciplinary Approaches to the Interpretation of Prehistoric and Historic Built Environments. De Gruyter. Berlin and Boston: 297-316. 
Stone, E. C. (1987): Nippur Neighbourhoods. Oriental Institute of the University of Chicago, Chicago.

Terpstra, D. (2014): Space and Neighbourhoods in Roman Ostia: The Spatial Organisation of Ostia's Insula IV iv and its Relation to the Larger City (unpublished MA Thesis). University of Leiden. Leiden.

van Nes, A. (2011): »Measuring spatial visibility, adjacency, permeability and degrees of street life in Pompeii«. En Laurence, R. and Newsome, D. J.
(Ed.): Rome, Ostia and Pompeii: Movement and Space,. Oxford University Press. Oxford: 100-118.

Vaughan, L., Jones, C. E., Griffiths, S., y Haklay, M. (2010): »The spatial signature of suburban town centres«. The Journal of Space Syntax. 1 (1): 77-91.

Wallace-Hadrill, A. (1994): Houses and Society in Prompeii and Herculaneum. Princeton University Press. Princeton. 